\title{
Impact of Lifestyle Intervention on the Estimated Visceral Fat Area and Skeletal Muscle Mass in Men with Obesity
}

\author{
Ali Madi Almajwal \\ Community Health Sciences Department, Clinical Nutrition Program, CAMS, King Saud University, Riyadh, KSA \\ Email: aalmajwal123@yahoo.com, aalmajwal@ksu.edu.sa
}

How to cite this paper: Almajwal, A.M. (2018) Impact of Lifestyle Intervention on the Estimated Visceral Fat Area and Skeletal Muscle Mass in Men with Obesity. Food and Nutrition Sciences, 9, 1434-1443. https://doi.org/10.4236/fns.2018.912104

Received: October 14, 2018

Accepted: December 25, 2018

Published: December 28, 2018

Copyright $\odot 2018$ by author and Scientific Research Publishing Inc. This work is licensed under the Creative Commons Attribution International License (CC BY 4.0).

http://creativecommons.org/licenses/by/4.0/

\section{(c) (i) Open Access}

\begin{abstract}
Background: The lifestyle modification remains the fundamental approach for the obesity treatment. The aim of this study is to demonstrate the effect of the multimodal lifestyle intervention on the estimated visceral fat area versus changes in musculoskeletal mass in a cohort of adult men with obesity. Subjects and Methods: A retrospective cohort study in which the file of eighty-two male subjects, aged 20 - 60 years, was studied for three months. Patients had been instructed to follow a balanced-hypocaloric diet, physical activity plan and general advice for combating the unhealthy lifestyle habits through the study period. Those who succeeded to loss $>5 \%$ of their body weight were classified as weight loser (WL) group, while others as weight resistant (WR) group. The results of In Body-720 bioelectric impedance analysis were used to report the fat mass (FM), visceral fat area (VFA), skeletal muscle mass (SMM), SMM/VFA ratio, osseous mass (OM), and basal metabolic rate (BMR) before and after the study period. Anthropometric measures, glucose, and lipid profile were also analyzed. Paired t-test was used to detect the significance of change between before and after measures, independent sample t-test was used to compare WL vs. WR groups. Results: There were significant decreases in weight, FM $(p<0.05)$ and VFA $(p<$ $0.001)$, in addition to a significant rise of SMM/VFA ratio $(p<0.05)$, together with insignificant changes of osseous mass, SMM and BMR after 3 months. Additionally, total cholesterol, low-density lipoprotein, and triglycerides were significantly reduced $(P<0.05)$. The percentages of reduction in weight, FM and VFA in WL vs. WR groups were highly significant $(p<0.001)$, while the percentages of change in SMM, SMM/VFA ratio and BMR were significant ( $p$ $<0.05)$. Conclusion: Lifestyle modification might be effective in production of positive changes in the body composition of patients with obesity. These changes were more significant in weight losers.
\end{abstract}




\section{Keywords}

Visceral Fat Area, Skeletal Muscle Mass, SMM/VFA Ratio, Obesity, InBody-720

\section{Introduction}

The wide prevalence of obesity and its association with many preventable comorbidities makes the methods to combat that obesity is essential. Obesity-managing protocols may shift early to pharmacological or surgical solutions to obesity in order to achieve rapid general weight loss [1]. Although recent studies confirm that obesity-related comorbidities are primarily developed as a result of exaggeration and disturbance of the visceral adipose tissue which in turn produces dysregulation of many adipokines [2] [3]. Accordingly, the main problem of obesity is the exaggerated and dysregulated visceral adipose tissue. So, reduction of the visceral fat during weight loss therapy is the main target, not merely a loss of total body weight [4].

The integrative lifestyle modification which includes a balanced hypocaloric diet, stepped engagement in a regular physical activity plan [7], and the behavioral modification in a gradual model to acquire a healthier lifestyle is the cornerstone in obesity management [5] [6].

Bioelectrical impedance analysis (BIA) is a non-invasive technique that widely used in the clinical settings for body composition analysis. The impedance of the measured body is calculated by assessing the resistance and reactance of tissues in the presence of a circuit of multifrequency alternating current i.e. it electrically quantifies the tissues for diagnostic purposes [7]. A lot of parameters could be given by a BIA test such as fat mass (FM), visceral fat area (VFA), fat free mass, skeletal muscle mass (SMM), SMM/VFA ratio, osseous mass (OM) in addition to prediction of basal metabolic rate (BMR). It was reported that the BIA-estimated visceral fat area (eVFA) is highly related and close to that measured by computed tomography (CT) [8]. SMM/VFA ratio was proved to be the main factor in the relationship of the adipo-myokines such as iris in hormone to its metabolic functions [9] and to the development of metabolic syndrome [10]. Accordingly, the aim of this study is to demonstrate the effect of the multimodal lifestyle intervention on the estimated visceral fat area versus changes in musculoskeletal mass in a cohort of adult men with obesity.

\section{Subjects and Methods}

Subjects: All participants were cases of the weight reduction clinic, therapeutic nutrition clinical services in the college of applied medical sciences (CAMS), male sector, King Saud University, between September 2016 to January 2017. Files of eighty-two adult individuals, 20 - 62 years old were selected to be used in 
a retrospective cohort study for three months. The sample size was calculated based on expected percent of weight loss about $10.8 \%$ [5], with a statistical power (1-beta, \% chance of detecting) of $80 \%$ and Two-sided significance level (1-alpha) of $95 \%$. The estimated sample size was 82 participants. Selected cases had been examined at the first-visit day (basal assessment), every biweekly appointment and at the end for three-months' period (the final assessment). Exclusion criteria included dehydrated or edematous patients, those having a pacemaker or a metal implant, diabetic or cancer patients, and those with severe disability, or psychiatric disturbance. Committed patients with a medically significant weight loss $(\geq 5 \%)$ in the three months' period were classified as weight loser (WL) group, while those who failed to lose up to $5 \%$ of their original weight in the same periods, despite of partial following of the lifestyle intervention, were classified as weight resistant (WR) group. According to the policy of the CAMS's clinical services, every case should sign an informed consent permitting the use of his data for research purposes. Furthermore, the research ethics committee of CAMS approved the study design and protocol.

Anthropometric measures: Values of the height, body weight, body mass index (BMI), and waist circumference (WC) were reported and used for analysis.

Lifestyle intervention: All cases were interviewed for forty minutes on the day one. The dietary, physical activity and lifestyle history of each subject were used to tailor a patient-centered lifestyle intervention program with some common features: a) Six-hundred calorie-deficit, however, balanced diet which was prescribed in form of three meals and two snacks in between. b) Physical activity regimen including basal daily activities such as daily walking, home activities...etc., for 30 minutes, in addition to the practice of aerobics in a moderate intensity such as gym activities, swimming...etc., for $60 \mathrm{~min}$ at least five days/week. c) Behavioral modification of unhealthy habits using a variety of problem-solving tools [5] [6].

Bioelectrical impedance analysis: Results of an eight-channel multifrequency bioelectrical impedance analysis (InBody 720; Biospace, Korea) were examined. VFA, SMM, FM, OM, fitness score, and BMR were recorded and used in statistical analysis. Values of SMM were divided on VFA to calculate SMM/VFA ratio [9]. Fitness score was based on comparison with the target values for ideal body fatness. Fitness score is calculated as a proportion between a given subject and a reference man with optimal muscle/fat ratio. Its values range from 0 to 100, while those values near 100 mean the best.

Biochemical analysis: Recorded results of fasting blood glucose and lipid panel which was done according to the previous methods [11] [12] were used in the statistical analysis.

Statistical analysis: SPSS for Windows (version 19; SPSS Inc., Chicago, IL, USA) was used for data analysis. Data were reported as the mean and standard deviation (SD). Paired t-test was used to compare measurements before and af- 
ter the intervention. Independent sample t-test was used to compare WL and WR groups. Pearson correlation coefficient was used to test the relation between SMM and VFA. 95\% confidence interval and $\mathrm{P} \leq 0.05$ were considered of statistical significance.

\section{Results}

The mean age of participants of this study was $34.25 \pm 14.42$ years. Demographic characteristics of the study cohort showed $85 \%$ as Saudi, $13.8 \%$ as Arabic nationalities and $1.2 \%$ as Asian participants. Educational level varies among secondary school, bachelor, and postgraduate level as $18.29 \%, 36.59 \%$ and $45.12 \%$, respectively. From a socioeconomic point of view, $41.46 \%$ had salaries between 10 to 20 thousand Saudi Riyals, in addition to a $41.46 \%$ below this range and $10.98 \%$ above.

Correlation of VFA with SMM (Figure 1) showed a significant moderate correlation $(r=0.398, p=0.010$ ), while the OM failed to be significantly correlated with the VFA.

As shown in Table 1, before-after comparison of this cohort revealed highly significant reductions of BMI and VFA $(p<0.001)$, as well as significant reductions of weight, WC, FM, total and LDL-cholesterol, and triglycerides levels $(p<$ $0.05)$. Furthermore, there were increase of SMM/VFA ratio $(p<0.05)$ and the fitness score $(p<0.001)$, together with insignificant changes of SMM, OM, BMR, glucose and HDL-cholesterol levels.

For WL group (72\% of all cases), the mean of percentages of weight loss, FM loss, and VFA loss after 3-months period were $10.87 \%, 23.08 \%$, and $23.17 \%$, respectively. SMM/VFA ratio increased by about $29.47 \%$, fitness score raised by $12.18 \%$. The reduction in $\mathrm{OM}$ and $\mathrm{BMR}$ were very minor (0.38\% \& $1.70 \%)$. The comparison of WL with WR groups showed significant differences in all percentages of change except the percent of the osseous mass loss (Table 2). Comparison of the demographic data between the two groups was presented in Table 3. Generally, weight resistance was common in low educational and socioeconomic status.

\section{Discussion}

Normalization of the lifestyle of patients with obesity or overweightedness by calorie-restricted and nutrients-complete diet, physical activity plan and acquisition of a healthier behavior, might improve the body composition and may provide a considerable method for management of obesity [13].

This report showed that intensive and integrated lifestyle modification which included dietary regimen, physical activity and stopping of unhealthy dietary habits, (i.e. 3PP intervention) might be effective in loss of the exaggerated visceral adipose tissue. Despite minor body weight loss, the reduction of the estimated visceral fat area and the mass of the fat compartment were promising. In addition, the changes in BMI (as a general indicator of obesity), and WC (as a crude indicator of the central obesity) were less significant than the percent of 


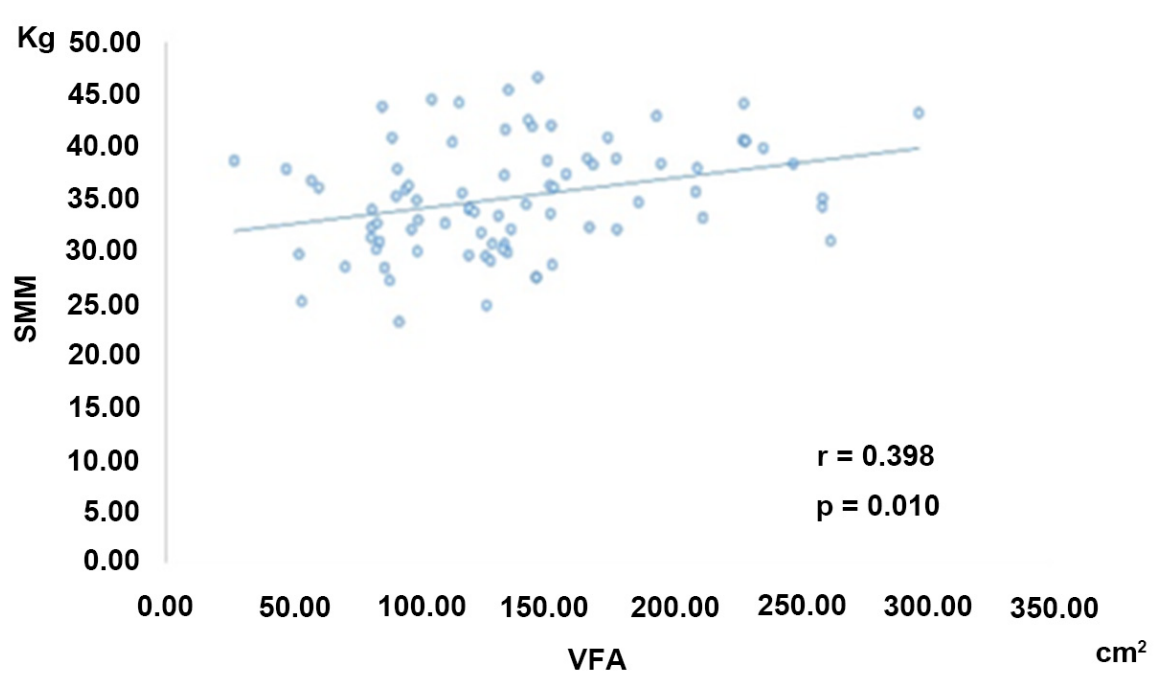

Figure 1. Correlation of the skeletal muscle mass (SMM) with visceral fat area (VFA).

Table 1. Measured variables before and after 3 months of lifestyle intervention $(n=82)$.

\begin{tabular}{|c|c|c|c|}
\hline \multirow{2}{*}{ Parameters } & \multicolumn{2}{|c|}{ Means \pm SD } & \multirow{2}{*}{$P$-value } \\
\hline & Before & After & \\
\hline Weight $(\mathrm{kg})$ & $98.81 \pm 20.59$ & $93.44 \pm 21.11$ & $<0.05^{*}$ \\
\hline BMI $\left(\mathrm{kg} / \mathrm{m}^{2}\right)$ & $33.52 \pm 6.84$ & $31.76 \pm 6.77$ & $<0.001^{*}$ \\
\hline $\mathrm{WC}(\mathrm{cm})$ & $116.67 \pm 14.43$ & $105.17 \pm 16.99$ & $<0.05^{*}$ \\
\hline $\mathrm{FM}(\mathrm{kg})$ & $37.87 \pm 12.91$ & $32.21 \pm 12.17$ & $<0.05^{*}$ \\
\hline VFA $\left(\mathrm{cm}^{2}\right)$ & $153.97 \pm 56.57$ & $127.90 \pm 56.37$ & $<0.001^{*}$ \\
\hline SMM (kg) & $35.09 \pm 5.21$ & $34.83 \pm 5.15$ & 0.279 \\
\hline SMM/VFA ratio & $0.26 \pm 0.10$ & $0.34 \pm 022$ & $<0.05^{*}$ \\
\hline $\mathrm{OM}(\mathrm{kg})$ & $3.50 \pm 0.54$ & $3.48 \pm 0.48$ & 0.651 \\
\hline Fitness score & $61.20 \pm 11.87$ & $65.33 \pm 11.60$ & $<0.001^{*}$ \\
\hline BMR(Kcal/day) & $1710.20 \pm 189.08$ & $1701.75 \pm 186.46$ & 0.316 \\
\hline Glucose (mg/dl) & $99.40 \pm 10.50$ & $103.60 \pm 10.69$ & 0.589 \\
\hline T. Chol. (mg/dl) & $213.14 \pm 37.57$ & $177.71 \pm 27.77$ & $<0.05^{*}$ \\
\hline $\mathrm{LDL}(\mathrm{mg} / \mathrm{dl})$ & $123.50 \pm 27.80$ & $99.67 \pm 33.09$ & $<0.05^{*}$ \\
\hline $\mathrm{HDL}(\mathrm{mg} / \mathrm{dl})$ & $47.60 \pm 8.96$ & $49.00 \pm 8.75$ & 0.683 \\
\hline $\mathrm{TG}(\mathrm{mg} / \mathrm{dl})$ & $135.00 \pm 37.12$ & $82.20 \pm 26.88$ & $<0.05^{*}$ \\
\hline
\end{tabular}

*Significant, WC; waist circumference, FM; fat mass, VFA; visceral fat area, SMM; skeletal muscle mass, OM; osseous mass, BMR; basal metabolic rate, T. Chol.; total cholesterol, and TG; triglycerides.

fat mass loss and the eVFA loss. In another study, dietary interventions without properly planned physical activity resulted in losing more than 5 percent of body weight after six months of hypocaloric diet [14]. Furthermore, nutritional education especially healthy eating education resulted in improvement of dietary intake among a sample of university students [15]. Taken together, it became 
Table 2. Percentages of the measured parameters' change in weight losers versus resistant groups.

\begin{tabular}{cccc}
\hline Parameters & $\begin{array}{c}\text { Weight losers } \\
(\mathrm{n}=59)\end{array}$ & $\begin{array}{c}\text { Weight resistant } \\
(\mathrm{n}=23)\end{array}$ & $P$-value \\
\hline \% Weight loss & $10.87 \pm 4.81$ & $1.49 \pm 1.56$ & $<0.001^{*}$ \\
\% BMI loss & $9.65 \pm 3.72$ & $1.89 \pm 1.72$ & $<0.001^{*}$ \\
\% FM loss & $23.08 \pm 8.19$ & $3.87 \pm 6.20$ & $<0.001^{\star}$ \\
\% VFA loss & $23.17 \pm 10.55$ & $6.53 \pm 11.53$ & $<0.001^{*}$ \\
\% SMM change & $2.51 \pm 3.05$ & $-0.91 \pm 3.38$ & $<0.05^{\star}$ \\
\% SMM/VFA ratio change & $-29.47 \pm 20.67$ & $-10.04 \pm 15.35$ & $<0.05^{\star}$ \\
\% Osseous mass change & $0.38 \pm 4.05$ & $-1.53 \pm 3.20$ & 0.122 \\
\% Fitness score change & $-12.18 \pm 9.20$ & $-3.87 \pm 5.84$ & $<0.05^{\star}$ \\
\% BMR change & $1.70 \pm 2.16$ & $-0.65 \pm 2.48$ & $<0.05^{\star}$ \\
\hline
\end{tabular}

NB: Negative values mean increase of these parameters, ${ }^{\star}$ Significant, FM; fat mass, VFA; visceral fat area, SMM; skeletal muscle mass, BMR; basal metabolic rate.

Table 3. Comparison of the demographic data between weight losers versus resistant groups.

\begin{tabular}{ccc}
\hline Parameters & $\begin{array}{c}\text { Weight losers } \\
(\mathrm{n}=59)\end{array}$ & $\begin{array}{c}\text { Weight resistant } \\
(\mathrm{n}=23)\end{array}$ \\
\hline Age & $27.25 \pm 7.85$ & $31.60 \pm 2.65$ \\
\hline Nationality & $32.21 \%$ & $47.82 \%$ \\
\hline Saudi & $49.15 \%$ & $30.44 \%$ \\
Arabic & $18.64 \%$ & $21.74 \%$ \\
\hline Educational level & & $47.83 \%$ \\
\hline Secondary school & $15.25 \%$ & $30.43 \%$ \\
Bachelor & $52.55 \%$ & $21.74 \%$ \\
\hline Socioeconomic & $32.20 \%$ & $30.43 \%$ \\
\hline < 20,000 & & $60.87 \%$ \\
\hline
\end{tabular}

clear that lifestyle interventions including diet, physical activity, and behavioral modification were much better than an individual modality of management. This theory was supported by a recent study [16] that demonstrated the effect of the behavioral therapy alone as a non-diet lifestyle program for admitted morbid obesity patients with psychological comorbidity. It was concluded that morbidly obese participants might achieve a significant reduction in weight and amelioration of psychological manifestations. Additionally, the patient-physician rela- 
tionship plays an essential role in the management of obesity [17]. On another hand, a randomized controlled trial at the primary health care level [18] showed that a hypocaloric diet of proper macronutrient composition in a combination of physical activity practice had limited impact on body weight in adolescents. The different clinical setting may give explanation of this discrepancy.

Estimated VFA by using bioelectric impedance analysis was studied by many investigators [19]. One Japanese study stated that the cutoff value for estimated visceral fat area at $100 \mathrm{~cm}^{2}$ is useful for diagnosis of cardiovascular risk factors associated with obesity [20]. This proved that lifestyle intervention could significantly reduce the VFA more than the total fat mass in addition to improvement of lipid panel among patients with obesity. This result was consistent with Nicklas et al., [21] who reported that weight loss among postmenopausal African-American and Caucasian women, caused by calorie restriction and practice of physical activities effectively reduce visceral fat area measured by computed tomography (CT). Considering the obesity degree, most of our cases were first-degree obesity and overweight (the mean basal BMI was $33.73 \pm 5.76$ $\mathrm{kg} / \mathrm{m}^{2}$ ). Thus, results of the current study could be considered as a continuation to a recent meta-analysis, which revealed that lifestyle modification trials including a physical activity could improve body weight and other cardiometabolic risk factors in the second and third degree of obesity [22].

In this study, cases were instructed to start low-intensity daily activities, and then gradually progress to a higher intensity. This pyramid-like progression in the intensity of the physical activity shared in the reduction of visceral adipose tissue. In contrary to this finding, Irving et al., [23] reported that only high-intensity exercise training could affect the visceral fat area among obese women. The gender difference may explain this discrepancy.

Furthermore, this intervention produced a partial improvement of lipid profile especially total cholesterol, LDL cholesterol, and triglycerides, while HDL insignificantly changed. Consistent with that, Unick et al., [24] stated that long-term intensive lifestyle intervention for severely obese patients produced a significant reduction in LDL, total cholesterol, triglycerides, glucose and blood pressure, but failed to produce favorable improvement of HDL cholesterol. On another hand, the results of the current study were not in line with the pediatric results of a multidisciplinary family-based lifestyle intervention which revealed insignificant effects on lipid profile. However, it significantly affects the adiposity, physical fitness and glucose homeostasis [25].

Despite of these positive results, this study has some limitations. The main limitations are the retrospective nature of this investigation and the use of BIA rather than the gold standard MRI.

\section{Conclusion}

In conclusion, the integrative multi-modality lifestyle intervention for obesity treatment might be a valuable method that reduces visceral fat area. Future re- 
search should investigate methods of augmentation of this effect and discover the underlying mechanisms.

\section{Conflicts of Interest}

The author declares no conflicts of interest regarding the publication of this paper.

\section{References}

[1] Apovian, C.M., Garvey, W.T. and Ryan, D.H. (2015) Challenging Obesity: Patient, Provider, and Expert Perspectives on the Roles of Available and Emerging Nonsurgical Therapies. Obesity, 23, S1-S26. https://doi.org/10.1002/oby.21140

[2] Fukuhara, A., Matsuda, M., Nishizawa, M., Segawa, K., Tanaka, M., Kishimoto, K., et al. (2005) Visfatin: A Protein Secreted by Visceral Fat That Mimics the Effects of Insulin. Science, 307, 426-430. https://doi.org/10.1126/science.1097243

[3] Gauvreau, D., Villeneuve, N., Deshaies, Y. and Cianflone, K. (2011) Novel Adipokines; Links between Obesity and Atherosclerosis. Annales d Endocrinologie, 72, 224-231. https://doi.org/10.1016/j.ando.2010.02.025

[4] Abulmeaty, M.M.A., Almajwal, A.M., Alsaif, M.A., Hassan, H.M. and Almansour, S.K. (2016) Impedancemetry vs. Anthropometry in Prediction of Body Adiposity and Obesity Diagnosis. Progress in Nutrition, 18, 39-46.

[5] Abulmeaty, M.M.A. (2016) Multimodal-Lifestyle Intervention Produces Reduction of the Fat Mass Rather than Body Weight Loss in Men with Obesity: A Prospective Cohort Study. Nutrition Clinique et Métabolisme, 30, 163-171. https://doi.org/10.1016/j.nupar.2016.04.001

[6] Foster, G.D., Makris, A.P. and Bailer, B.A. (2005) Behavioral Treatment of Obesity. American Journal of Clinical Nutrition, 82, 230S-235S. https://doi.org/10.1093/ajcn/82.1.230S

[7] Riera, J., Riu, P.J., Casan, P. and Masclans, J.R. (2011) Electrical Impedance Tomography in Acute Lung Injury. Medicina Intensiva, 35, 509-517. https://doi.org/10.1016/j.medin.2011.05.005

[8] Ryo, M., Maeda, K., Onda, T., Katashima, M., Okumiya, A., Nishida, M., et al. (2005) A New Simple Method for the Measurement of Visceral Fat Accumulation by Bioelectrical Impedance. Diabetes Care, 28, 451-453.

https://doi.org/10.2337/diacare.28.2.451

[9] Hwang, Y., Jeon, W.S., Park, C. and Youn, B. (2016) The Ratio of Skeletal Muscle Mass to Visceral Fat Area Is a Main Determinant Linking Circulating Irisin to $\mathrm{Me}-$ tabolic Phenotype. Cardiovascular Diabetology, 15, 9.

https://doi.org/10.1186/s12933-015-0319-8

[10] Kim, T.N., Park, M.S., Lim, K.I., Yang, S.J., Yoo, H.J., Kang, H.J., et al. (2011) Skeletal Muscle Mass to Visceral Fat Area Ratio Is Associated with Metabolic Syndrome and Arterial Stiffness: The Korean Sarcopenic Obesity Study (KSOS). Diabetes Research and Clinical Practice, 93, 285-291.

https://doi.org/10.1016/j.diabres.2011.06.013

[11] Panz, V.R., Raal, F.J., Paiker, J., Immelman, R. and Miles, H. (2005) Performance of the CardioChek PA and Cholestech LDX Point-of-Care Analysers Compared to Clinical Diagnostic Laboratory Methods for the Measurement of Lipids. Cardiovascular Journal of South Africa, 16, 112-119.

[12] Parikh, P., Mochari, H. and Mosca, L. (2009) Clinical Utility of a Finger Stick 
Technology to Identify Individuals with Abnormal Blood Lipids and High-Sensitivity C-Reactive Protein Levels. American Journal of Health Promotion, 23, 279-282. https://doi.org/10.4278/ajhp.071221140

[13] Minich, D.M. and Bland, J.S. (2013) Personalized Lifestyle Medicine: Relevance for Nutrition and Lifestyle Recommendations. Scientific World Journal, 2013, Article ID: 129841. https://doi.org/10.1155/2013/129841

[14] Elbelt, U., Schuetz, T., Knoll, N. and Burkert, S. (2015) Self-Directed Weight Loss Strategies: Energy Expenditure Due to Physical Activity Is Not Increased to Achieve Intended Weight Loss. Nutrients, 7, 5868-5888. https://doi.org/10.3390/nu7075256

[15] Shahril, M.R., Wan Dali, W.P. and Lua, P.L. (2013) A 10-Week Multimodal Nutrition Education Intervention Improves Dietary Intake among University Students: Cluster Randomised Controlled Trial. Journal of Nutrition and Metabolism, 2013, Article ID: 658642.

[16] Bannert, B., Schobersberger, W., Tran, U. and Remmel, A. (2011) The Effectiveness of a Non-Diet Multidisciplinary Weight Reduction Program for Severe Overweight Patients with Psychological Comorbidities. Journal of Obesity, 2011, Article ID: 641351. https://doi.org/10.1155/2011/641351

[17] Kiesewetter, S., Köpsel, A., Mai, K., Stroux, A., Bobbert, T., Spranger, J., et al. (2012) Attachment Style Contributes to the Outcome of a Multimodal Lifestyle Intervention. Bio Psycho Social Medicine, 6, 3-8. https://doi.org/10.1186/1751-0759-6-3

[18] Jansson, S.P.O., Engfeldt, P., Magnuson, A., Georg Lohse, P.T. and Liljegren, G. (2013) Interventions for Lifestyle Changes to Promote Weight Reduction, a Randomized Controlled Trial in Primary Health Care. BMC Research Notes, 6, 213-219. https://doi.org/10.1186/1756-0500-6-213

[19] Park, K.S., Lee, D.H., Lee, J., Kim, Y.J., Jung, K.Y., Kim, K.M., et al. (2016) Comparison between Two Methods of Bioelectrical Impedance Analyses for Accuracy in Measuring Abdominal Visceral Fat Area. Journal of Diabetic Complications, 30, 343-352. https://doi.org/10.1016/j.jdiacomp.2015.10.014

[20] Okauchi, Y., Kishida, K., Funahashi, T., Nogouchi, M., Ogawa, T., Ryo, M., et al. (2010) Absolute Value of Bioelectrical Impedance Analysis-Measured Visceral Fat Area with Obesity-Related Cardiovascular Risk Factors in Japanese Workers. Journal of Atherosclerosis and Thrombosis, 17, 1237-1245.

https://doi.org/10.5551/jat.5694

[21] Nicklas, B.J., Dennis, K.E., Berman, D.M., Sorkin, J., Ryan, A.S. and Goldberg, A.P. (2003) Lifestyle Intervention of Hypocaloric Dieting and Walking Reduces Abdominal Obesity and Improves Coronary Heart Disease Risk Factors in Obese, Postmenopausal, African-American and Caucasian Women. The Journals of Gerontology. Series A, Biological Sciences and Medical Sciences, 58, 181-190. https://doi.org/10.1093/gerona/58.2.M181

[22] Baillot, A., Romain, A.J., Boisvert-Vigneault, K., Audet, M., Baillargeon, J.P., Dionne, I.J., et al. (2015) Effects of Lifestyle Interventions That Include a Physical Activity Component in Class II and III Obese Individuals: A Systematic Review and Meta-Analysis. PLoS ONE, 10, e0119017. https://doi.org/10.1371/journal.pone.0119017

[23] Irving, B.A., Davis, C.K., Brock, D.W., Weltman, J.W., Swift, D., Barrett, E.J., et al. (2008) Effect of Exercise Training Intensity on Abdominal Visceral Fat and Body Composition. Medicine \& Science in Sports \& Exercise, 40, 1863-1872. https://doi.org/10.1249/MSS.0b013e3181801d40

[24] Unick, J.L., Beavers, D., Bond, D.S., Clark, J.M., Jakicic, J.M., Kitabchi, A.E., et al. 
(2013) The Long-Term Effectiveness of a Lifestyle Intervention in Severely Obese Individuals. The American Journal of Medicine, 126, 236-242.

https://doi.org/10.1016/j.amjmed.2012.10.010

[25] Vos, R.C., Wit, J.M. and Houdijk, E.C.A.M. (2011) Long-Term Effect of Lifestyle Intervention on Adiposity, Metabolic Parameters, Inflammation and Physical Fitness in Obese Children: A Randomized Controlled Trial. Nutrition \& Diabetes, 1 , e9. https://doi.org/10.1038/nutd.2011.5 